\title{
Rehabilitación de maxilares clases I y II de Kennedy mediante prótesis removibles
}

\section{Rehabilitation of maxillary Kennedy class I \& II treated with removable dentures}

\author{
Enrique Fernández ${ }^{1 *}$, Hernán Acosta ${ }^{2}$, Ignacio Madrid ${ }^{3}$, Luis Verdugo ${ }^{2}$
}

\begin{abstract}
1. Cirujano Dentista, Universidad de Chile, Especialista en Rehabilitación Oral, Profesor Asistente y Docente de Clínicas Integradas y del Post Grado de Rehabilitación Oral, Facultad de Odontología, Universidad San Sebastián, CHILE.

2 Cirujano Dentista, Universidad San Sebastián, Docente Clínicas Integradas, Facultad de Odontología, Universidad San Sebastián, CHILE. 3 Alumnos V año Clínicas Integradas, Facultad de Odontología, Universidad San Sebastián, CHILE.
\end{abstract}

${ }^{*}$ Correspondencia a: Prof.Dr. Enrique Fernández M. Facultad de Odontología, Universidad San Sebastián. Av. Ricardo Cumming \#40, CP 8320000, Santiago, CHILE. | e-mail: enrique.fernandez@uss.cl

Trabajo recibido el 29/03/2016. Aprobado para su publicación el 26/11/2016

\begin{abstract}
RESUMEN
La irrupción de los implantes óseo integrados y su posterior validación, a comienzos de los años '80, aportó sin duda un utilísimo recurso en la rehabilitación oral. Lamentablemente, en gran medida debido a los costes económicos involucrados, a pesar de alentadores augurios, no han devenido en una alternativa terapéutica concreta para la gran mayoría de los pacientes desdentados. El edentulismo parcial de maxilas, clases Kennedy I y II, con remanencia de todos o parte de los dientes del sextante 2, en su mayoría casos de sustancial complejidad, léase clases III del Índice Diagnóstico Prostodóntico, genera un desafío protésico a solventar en relación a la retención y satisfacción estética de los pacientes. Presentamos en este reporte dos de estos casos, rehabilitados mediante prótesis metálicas "convencionales", en los cuales creemos logramos conjugar acertadamente ambas variables, combinando complejos retentivos RMC y facetas retentivas friccionales.
\end{abstract}

\section{PALABRAS CLAVE}

Prótesis removible; Clases de Kennedy; Índice diagnóstico prostodóntico; Complejo retentivo RMC

Rev. Clin. Periodoncia Implantol. Rehabil. Oral Vol. 10(2); 96-100, 2017.

\begin{abstract}
The emergence of osseointegrated implants and their further validation, at the beginning of the 80 's, contributed undoubtedly to oral rehabilitation as a very useful resource. Unfortunately, mainly because of the economic costs involved and despite an encouraging initial augury, they have not become in a specific therapeutic alternative for the vast majority of edentulous patients. Partial edentulism of maxillae, Kennedy's class I, \& II, with all or a few teeth remaining at the sextant 2 , mostly class III PDI, substantially compromised denture-supporting anatomy, generate a prosthetic challenge to overcome regarding retention and aesthetic satisfaction of these patients. In this report, we display two of them, which we believe have been successfully managed in order to fulfill both variables, by means of "conventional" removable metallic prostheses combining MGR's clasps and frictional retentive facets.

KEYWORDS

Removable prostheses; Kennedy classes; Prosthodontic diagnostic index; MGR clasp assembly
\end{abstract}

Rev. Clin. Periodoncia Implantol. Rehabil. Oral Vol. 10(2); 96-100, 2017.

\section{INTRODUCCIÓN}

Pese a todos los avances que la Odontología ha efectuado, especialmente en el área de la prevención y de manera sinérgica con la inversión de la pirámide demográfica, lo concreto es que permanece ampliamente vigente la necesidad de rehabilitar desdentaciones en una gran cantidad de población mundial, mediante procedimientos protésicos "convencionales". Esta situación se verifica indistintamente en países desarrollados así como en aquellos en "vías de desarrollo" $(1,2,3,4)$.

En esta perspectiva, siempre que nos enfrentamos a maxilas y mandíbulas parcialmente desdentadas, clasificadas topográficamente como clases I y II de Kennedy, surge una dificultad adicional en los casos de las primeras, más aún cuando el paciente sólo conserva el grupo antero superior o sextante(2). La situación se complejiza aún más cuando incluso faltan dientes en este sextante o bien permanece un solo remanente dentario en sextantes posteriores. De acuerdo con el Índice de Diagnóstico Prostodóntico (IDP) para pacientes parcialmente edéntulos, nuestros casos se tipifican como clase III, es decir de sustancial complejidad, en función de los criterios diagnósticos encontrados, que explicitaremos en relación a cada caso(5).

En las situaciones clínicas que expondremos a continuación, la fuerza de gravedad, en concomitancia con la exigencia estética, complica en mayor grado la obtención de una buena retención funcional de estas prótesis. En ambas intentamos, mediante la indicación en el sextante anterior de complejos retentivos RMC (MGR en inglés) $)^{(6)}$, en conjunto con Facetas Retentivas Friccionales (FRF) ${ }^{(7)}$, conseguir la máxima retención y estética posibles.

Se deja constancia que los dos pacientes involucrados, firmaron el pertinente consentimiento informado. 


\section{CASO CLÍNICO 1}

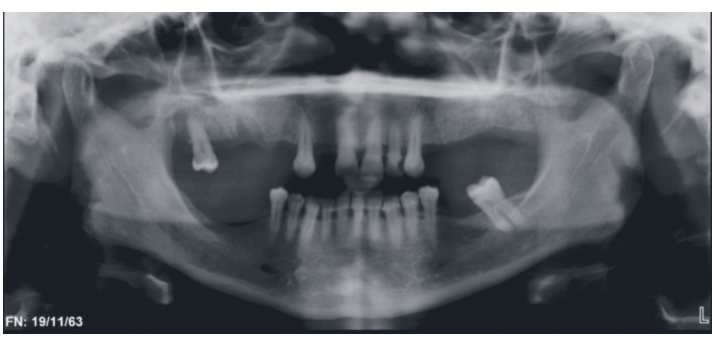

Figura 3. RX Panorámica caso 1

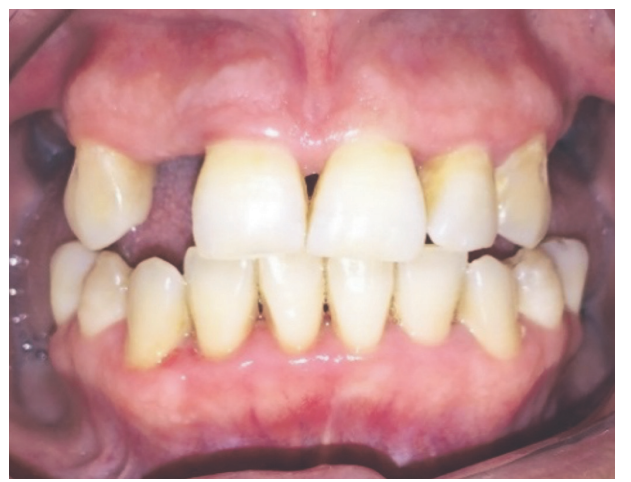

Figura 4. Caso 1, vista frontal, IDP clase III.

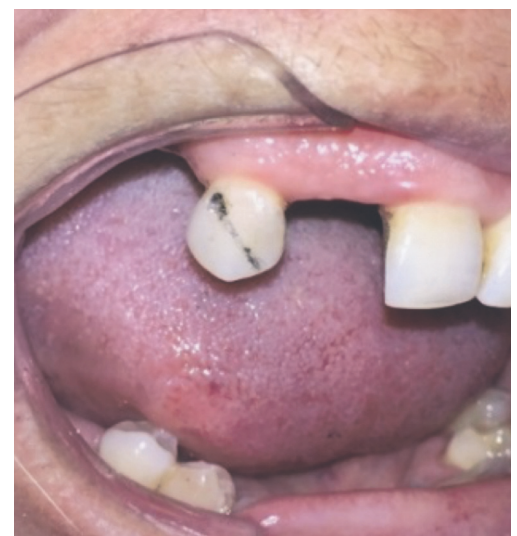

Figura 5. Planimetría de la oquedad vestibular diente 1.3

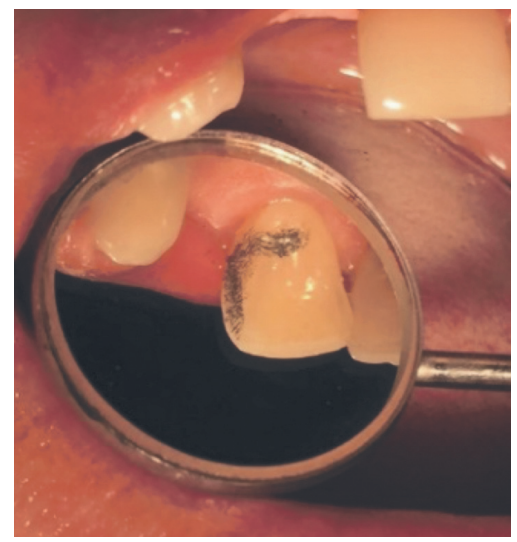

Figura 6. Planimetría de FRF, diente 1.1

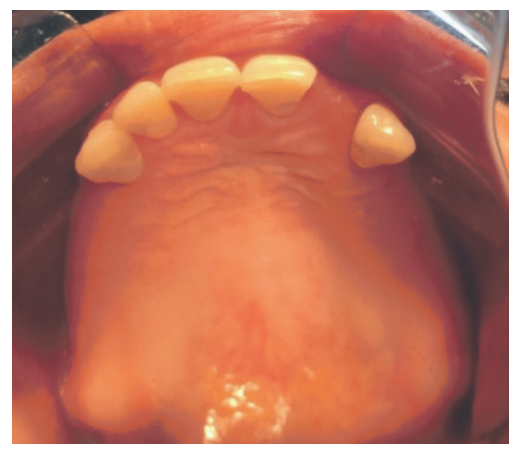

Figura 7. Caso 1, Vista oclusal, clase Kennedy I subdivisión 1.

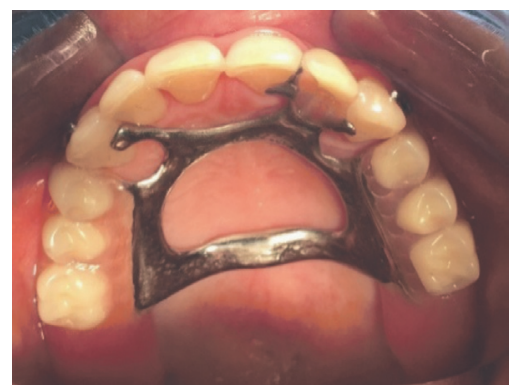

$\overline{\text { Figura 8. Caso 1, complejos retentivos RMC dientes 1.3, 2.3, FRF diente 1.1 }}$

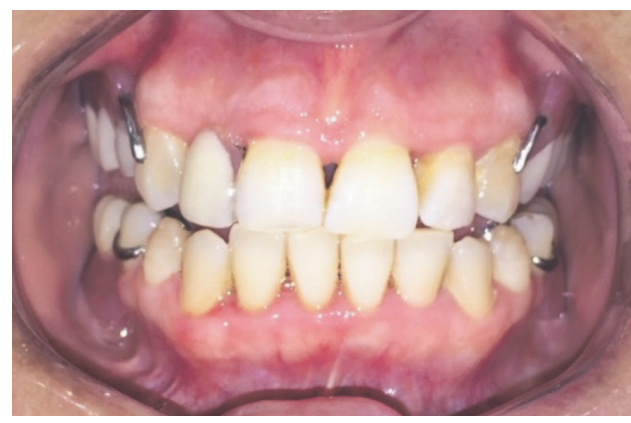

Figura 9. Caso 1, brazo retentivo en "I" RMC diente 1.3, 2.3

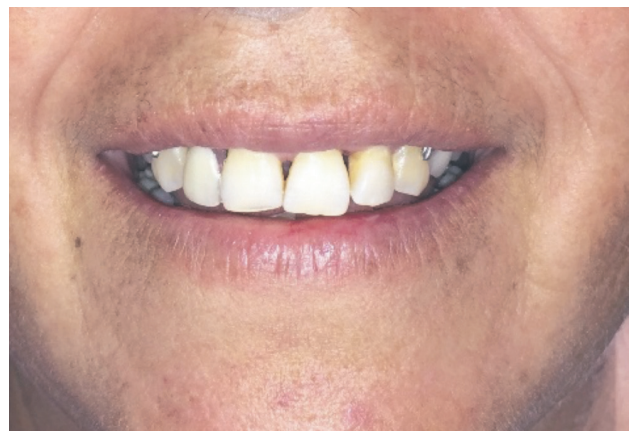

Figura 10. Caso 1, línea labial media "resultado estético".

Paciente 51 años, género femenino, HTA controlada, ASA II.

Soporte oclusal posterior ausente, maxilar clase I de Kennedy, subdivisión 1 (Fig. 7), mandíbula clase I de Kennedy áreas edéntulas posteriores de más de tres dientes, en ambas arcadas. Overjet y overbite, $2 \mathrm{~mm}$., guía anterior 1.1 y $2.1 / 3.1-3.2$ y $4.1-4.2$ ), con DVO mantenida por sextantes 2 y 5 . Presencia de sacos periodontales y caries.

Diagnóstico clínico: Periodontitis crónica generalizada severa inducida por placa bacteriana y enfermedad de caries.

Clasificación IDP: Clase III, demanda estética del paciente, sustancialmente comprometido(5).

Plan de tratamiento: A) Fase sistémica: Previos a procedimientos dentales invasivos, control de la presión arterial. B) Fase etiológica: Terapia periodontal, destartraje, pulidos coronarios y radiculares, reevaluación periodontal 21 días. C) Fase correctiva: Exodoncias 1.8 y 3.7. D) Fase rehabilitadora: Montaje de modelos de estudio y análisis 
en tangenciógrafo. Operatoria, resinas compuestas 1.3, 2.1, 2.2, 4.1 y 4.4. Preparación de la boca con fines protésicos: complejos retentivos RMC, dientes 1.3 (Fig. 5). y 2.3. Confección de oquedad o nicho en disto cérvico vestibular y lecho con ranura mesio palatina en ambos caninos. Complementa, FRF en distal diente 1.1 con lecho cingular palatino (Fig. 6). En mandíbula, tallado planos guías y lechos oclusales mesiales 3.5 y 4.5. Complejos retentivos de acción posterior en ambos pilares, retenedores anti rotacionales 3.3 y 4.3 , conector mayor barra lingual. Restablecimiento de la mesa oclusal, sin cambio de la DVO. E) Fase mantención: Reevaluación periodontal cada 3 meses y chequeo de fase rehabilitadora se aconseja cada 6 meses.

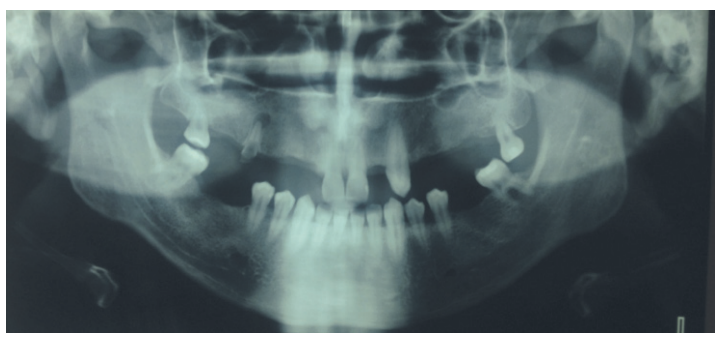

Figura 11. RX Panorámica caso 2

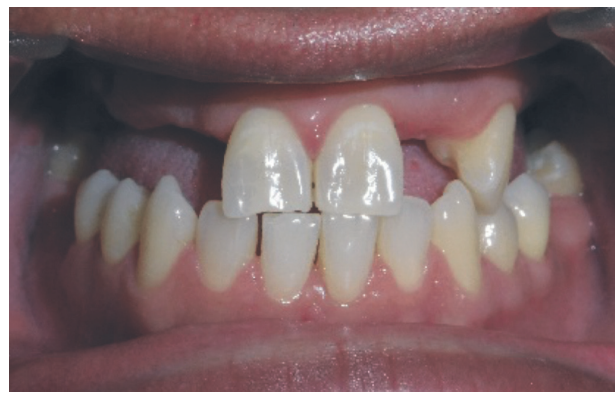

Figura 12. Caso 2, vista frontal, IDP clase III.

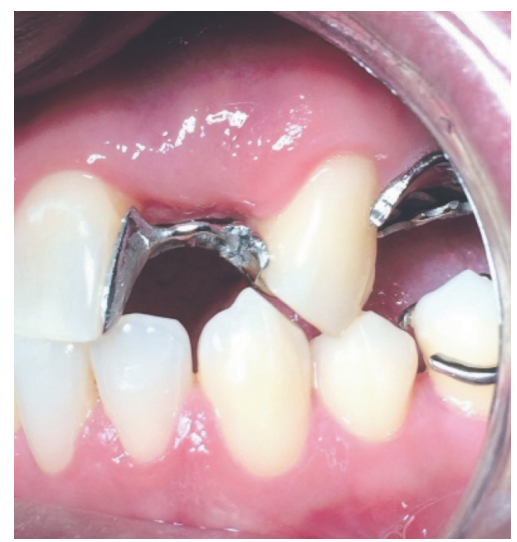

Figura 13. Caso 2, diente 2.1 FRF, diente 2.3 RMC

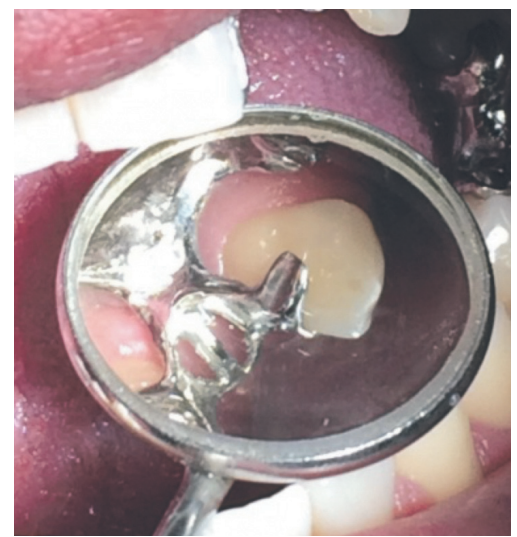

Figura 14. Caso 2, ranura y apoyo mesial diente 2.3

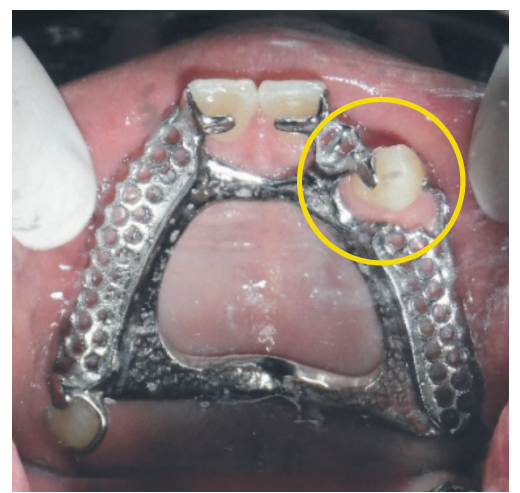

Figura 15. Caso 2, Zona disto cervical diente 2.3 sin cubrir

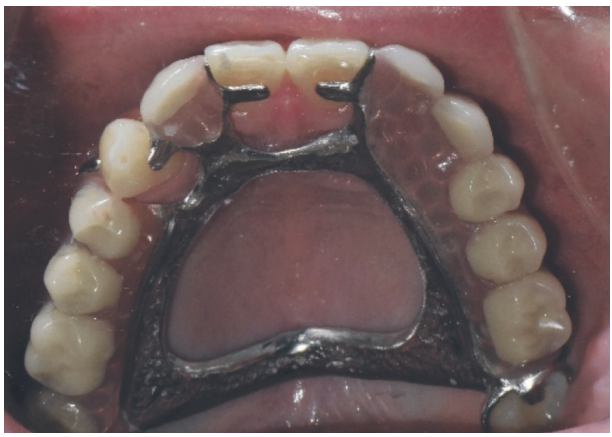

Figura 16. Caso 2, complejo retentivo RMC diente 2.3 y FRF dientes $1.1-2.1$

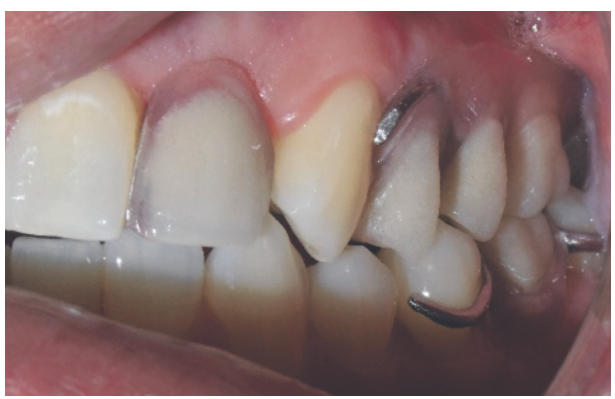

Figura 17. Caso 2, vista frontal complejo retentivo RMC diente 2.3 y FRF diente 2.1

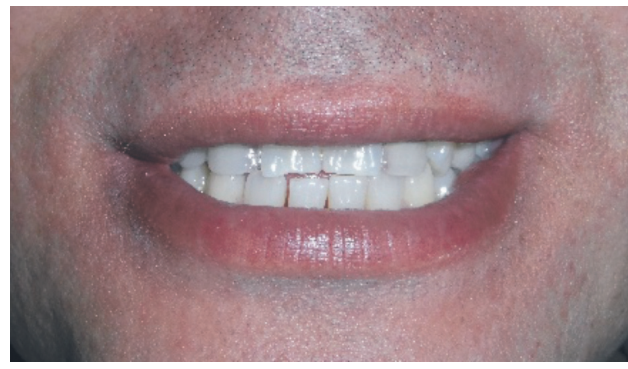

Figura 18. Caso 2, línea labial media, "resultado estético".

\section{CASO CLÍNICO 2}

Paciente 33 años, género masculino, Fumador, ASA I.

Soporte oclusal presente, maxilar Clase II de Kennedy, subdivisión 2 (Fig. 15), mandíbula Clase III de Kennedy, subdivisión 1. Relación maxilo mandibular clase III de Angle, overjet y overbite no observable, contacto vis a vis 1.1 y $2.1 / 3.1$ y 4.1 , con DVO mantenida. Presencia de sacos periodontales y caries. 
Diagnóstico clínico: Periodontitis crónica generalizada moderada modificada por tabaco y enfermedad de caries.

Clasificación IDP: Clase III, demanda estética del paciente, sustancialmente comprometido(5).

Plan de tratamiento: A) Fase sistémica: Sin intervención. B) Fase etiológica: Consejería cesación consumo tabaco; terapia periodontal, destartraje, pulidos coronarios y radiculares, exodoncia remanente radicular diente 1.5 no tratado con lesión apical (Fig. 11), reevaluación periodontal 21 días. C) Fase correctiva: Exodoncia diente 2.8 eventual pilar, desfavorable por raíz fusiforme con reabsorción ósea avanzada (Fig. 11). D) Fase rehabilitadora: Montaje de modelos de estudio y análisis en tangenciógrafo. Operatoria, resinas compuestas 3.4, 3.5 y 4.3. Preparación de la boca con fines protésicos: en maxilar, complejo retentivo RMC en diente 2.3, previa confección de oquedad o nicho en disto cérvico vestibular y lecho con ranura en mesio palatino (Fig. 14). FRF en distal diente 1.1 y 2.1 con lechos cingulares en palatino (Figs. 13 y 15). Complementa complejo retentivo de Akers en 1.8. En mandíbula, tallado planos guías y lechos oclusales dientes 3.5, 3.7, 4.5 y 4.7. Complejos retentivos de Ackers en los cuatro pilares, conector mayor barra lingual. E) Fase mantención: Reevaluación periodontal cada 3 meses y chequeo de fase rehabilitadora se aconseja cada 6 meses.

\section{DISCUSIÓN}

El Colegio Americano de Prostodoncistas (CAP) ha desarrollado un sistema de clasificación del edentulismo parcial, que desde el año 2005 fue incorporado al "Glosario de Términos Prostodónticos", sustentado por dicha asociación profesional(8). De hecho actualmente, todos los artículos clínicos sometidos a consideración editorial en la revista de este Colegio (Journal of Prosthodontics) deben presentar la clasificación de sus pacientes de acuerdo al IDP(9). El sistema de clasificación es similar al ya utilizado para el edentulismo completo, también elaborado por el CAP(10). El propósito de este sistema de clasificación es proveer de un marco clínico teórico de mayor coherencia para una planificación de acuerdo con las características del paciente y por ende, servir de guía para canalizar su necesidad de tratamiento prostodóntico con el nivel de especialización que requiriese ${ }^{(9)}$.

El cuestionario determinado por el ACP se basa en criterios de diagnóstico específicos y objetivos presentes en el examen inicial: condición de los dientes pilares, esquema oclusal, características del reborde residual, localización y extensión de las áreas edéntulas. Se definen entonces, cuatro categorías o clases de edentulismo parcial: clase I que representa una situación clínica no complicada o favorable, clase II y clase III con compromiso moderado y sustancial respectivamente y clase IV que representa una situación clínica compleja ${ }^{(5)}$.

En ambos pacientes de este reporte, como lo explicitamos, nos enfrentamos a clases III del IDP, atestiguando la complejidad inherente a la situación clínica abordada, más aún si se debe rehabilitar maxilas con recursos terapéuticos convencionales, verbigracia, prótesis parciales removibles metálicas.

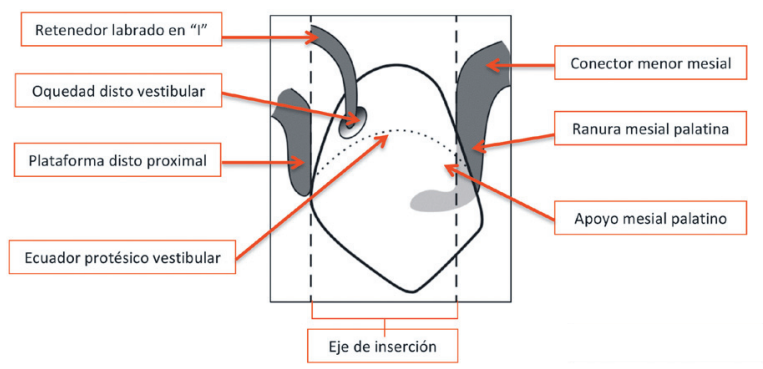

Figura 1. Esquema MGR original.

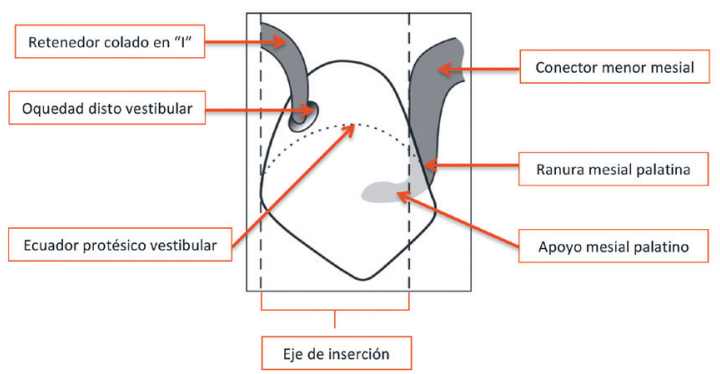

Figura 2. Esquema MGR modificado.
Aunque el factor estético no sea un imperativo o constituya un estándar en prótesis parcial removible ${ }^{(11)}$, para muchos pacientes la exposición de algún componente del complejo retentivo, en general brazos retentivos vestibulares, no resulta tolerable ${ }^{(11)}$. Más aún, para Mc Garry ${ }^{(5)}$, si existe una preocupación o desafío de orden estético de parte del paciente, entonces el grado de complejidad en el IDP se incrementa a un nivel inmediatamente superior, cuando se trata de Clases I y II de ese Índice.

Es así como con cierta frecuencia vemos en la literatura pertinente, incluso antes de la propuesta del MGR de Mc Cartney de $1981^{(6)}$, la presentación de complejos retentivos que pretenden superar esta falencia, incluso con diseños para incisivos laterales que deban "soportar" el emplazamiento de ese componente de la prótesis removible ${ }^{(11,12)}$.

Este es el concepto de función con estética, que subyace a la indicación y confección de FRF, motivando la generación de un artículo publicado en 2013. En él, destacábamos que las facetas se contra indicaban en clases I y II de Kennedy maxilares, puesto que no aseguraban suficiente retención a las prótesis en cuestión ${ }^{(7)}$.

Sin embargo, preparar FRF en otros dientes del sextante 2, de modo complementario al emplazamiento de un particular tipo de complejo retentivo en caninos, en estos casos el RMC, significó una alianza provechosa.

La concepción original del complejo retentivo con ranura mesial para la contención o reciprocidad (MGR en su denominación original en inglés: mesial groove reciprocation clasp), pretende dejar en cierta manera "encajado" al canino, para así aumentar al máximo su potencial de retención. Para dicho efecto, se emplaza el terminal de un brazo retentivo corto, de acceso cervical en forma de "I", en un desgaste u oquedad labrado en disto vestibular, cautelando también en lo posible el aspecto estético. En el diseño de Mc Cartney ${ }^{(6)}$, se confecciona con alambre de $0,8 \mathrm{~mm}$.(Fig. 1), pero nosotros introdujimos una primera modificación y lo indicamos colado con un corto recorrido vertical emergiendo desde el componente o brazo horizontal ${ }^{(13)}$. En una posición diagonalmente opuesta, mantenemos la confección del lecho y ranura en la cara mesio palatina (Fig. 2).

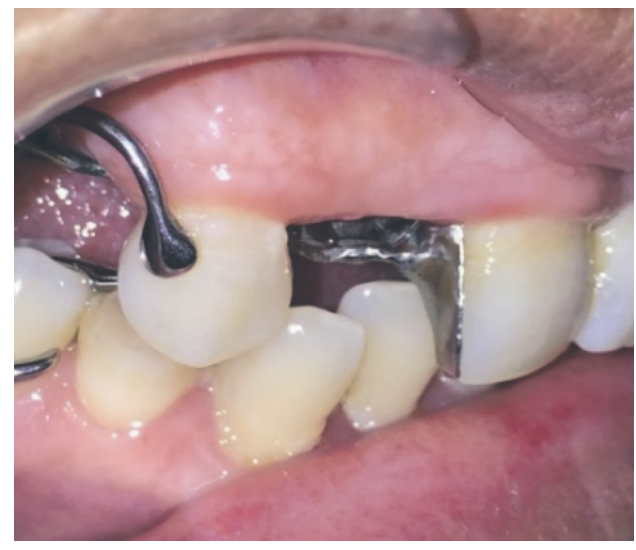

Figura 19. Nótese la dificultad para lograr de parte del laboratorio dental, un terminal retentivo en "l" alojado dentro del respectivo nicho y no una suerte de espátula emplazada sobre la oquedad.

La eventual excesiva rigidez de este complejo retentivo, debe a nuestro entender soslayarse, puesto que se está en presencia de un caso "límite" en cuanto a los recursos de que disponemos para que la prótesis tenga una mínima retención funcional y además, porque sabemos empíricamente que la mayor o más relevante agresión que puede resultar biomecanicamente perjudicial para un diente, proviene de la periodontitis y su potencial resultado de reabsorción ósea alveolar ${ }^{(14,15)}$.

También hemos generado otra modificación, eliminando la plataforma disto proximal del diseño original. Creemos que con los componentes presentes, el complejo retentivo presenta adecuada circunscripción y contención para su brazo activo, toda vez que despejamos la encía libre del margen cérvico distal del pilar. De este modo, evitamos favorecer retención de alimentos en esa zona, sustrato para generación de biofilm dental y por ende, promotor de enfermedad periodontal en los dientes pilares en cuestión ${ }^{(16,17)}$.

En este misma orden de cosas, cabe señalar que si bien la retención de estas prótesis removibles descansa esencialmente en la capacidad físico mecánica de sus componentes, tanto de los RMC como de las FRF, no se debe descartar cierta "complementariedad" con principios de retención muscular, propios de la prótesis total maxilar, 
en especial si nos aplicamos convenientemente en la obtención de un adecuado ajuste de los flancos de la prótesis en relación a las inserciones de los músculos para-protéticos y el pertinente alivio de los frenillos laterales ${ }^{(18)}$. Adicionalmente, la presencia de un área de soporte mayor constituida por la zona del paladar, permite una vía de transmisión mixta de cargas masticatorias, favorable para los pilares protésicos ${ }^{(19)}$.

Finalmente, un tópico no menor a destacar, en torno a estas rehabilitaciones mediante prótesis metálicas que escapan a lo convencional, es la dificultad que se nos presenta para que el técnico dental logre confeccionar adecuada y certeramente lo indicado por nosotros, en la transcripción gráfica al modelo de trabajo, del denominado diseño protésico ${ }^{(12,20)}$.

Al respecto, hay dos aspectos críticos a tener en consideración:

- La forma y ahusamiento que debe tener el terminal retentivo vestibular del RMC para lograr "acceder" al nicho u oquedad labrado allí para tal fin y conseguir el efecto retentivo planificado.

- Obtención de un punto o zona de contacto entre diente protésico y diente remanente que no deje percibir visualmente el metal de la FRF, perdiendo parte de la ventaja estética buscada con este artificio.

\section{CONCLUSIONES}

La rehabilitación de la desdentación parcial mediante prótesis metálicas o esqueléticas, continúa siendo requerida de manera intensiva alrededor del mundo, sin discriminar países con diferentes niveles de desarrollo socio económico ${ }^{(1,9,10)}$. Para algunos autores, en el tercer milenio aun parece razonable proponer este tipo de terapeútica y por tanto, no debe ser considerada obsoleta ${ }^{(21)}$. Por tal motivo, es menester perseverar en la actualización que le corresponde indelegablemente al odontólogo, tanto en la indicación como en los pasos clínicos conducentes a la correcta elaboración de la mejor prótesis posible para la satisfacción del paciente ${ }^{(3)}$.

Hemos presentado dos casos de sustancial complejidad clase III IDP, en los cuales complementar RMC en caninos, con FRF en otros remanentes, nos permitió entregar una buena solución a nuestros pacientes, habida consideración económica, con resultados funcionales y estéticos más que aceptables.

Sin embargo, tomando en cuenta la escasa relevancia cuantitativa constituida por dos casos, así como la muy reciente puesta en servicio de estos aparatos protésicos, es importante subrayar la ineludible tarea de llevar a cabo controles periódicos, periodicidad de 6 meses es la aconsejable, para asegurarnos que estas propuestas de diseño protésico tengan la sustentabilidad temporal y fisiológica que permita indicarlas certeramente como una solución a nuestros pacientes.

\section{Bibliográfia}

1. Carlsson GE, Omar R. The future of complete dentures in oral rehabilitation. A critical review. J Oral Rehabil 2010; 37:143-156.

2. Carlsson GE. Some dogmas related to prosthodontics, temporomandibular disorders and occlusion. Acta Odontol Scand 2010; 68:313-322.

3. Montero J, Castillo-Oyagu R, Lynch CH D, Albaladejo A, Castaño A. Selfperceived changes in oral health-related quality of life after receiving different types of conventional prosthetic treatments: A cohort follow-up study. J Dent 2013 41; 493-503.

4. Donovan TE, Derbabian K, Kaneko L, Wright TR. Esthetic considerations in removable prosthodontics. J Esthet Restor Dent 2001: 13:241-253.

5. McGarry TJ, Nimmo A, Skiba JF, Ahlstrom RH, Smith CR, et al. Classification system for partial edentulism. J Prosthodont 2002 Sep; 11 (3), 181-93.

6. Mc Cartney JW. The MGR clasp: An esthetic extracoronal retainer for maxillary canines. J Prosthet Dent 1981; 46(5):490-493.

7. Fernández E et al. Modificaciones de diseño protésico para mejorar estética en prótesis parcial removible metálica. Av. Odontoestomatol 2013; 29 (4): 175-183.

8. Felton DA. Exactly what is the hold up? J Prosthodont 2008; 17: 515-516.

9. Mahmood WA, Salim SA, Saharudin S. The status of the abutment teeth in distal extension removable partial dentures. Malay Dent J. $200930 ;(1)$ 13-19

10. Mazurat RD, Mazurat NM. Communicating complexity: using a diagnostic classification system for edentulous patients. J Can Dent Assoc 2003; 69: 511-514.

11. Khan SB, Geerts GAVM. Aesthetic clasp design for removable partial dentures: A literature review. SADJ 2005; 60: 190-194.

12. Tran $\mathrm{CH}$, LaBarre $\mathrm{E}$, Landesman $\mathrm{HM}$. A removable partial denture using an esthetically designed round-rest distal clasp on maxillary anterior abutment teeth: $A$ clinical report. J Prosthet Dent 2009; 102:286-289.

13. Sato $Y$, Tsuga K, Abe Y, Asahara S, Akagawa Y. Analysis of stiffness and stress in I-bar clasps. J Oral Rehabil 2001; 28:596-600.

14. Kern M, Wagner B. Periodontal findings in patients 10 years after insertion of removable partial dentures. J Oral Rehabil 2001 28; 991-997.

15. Akaltan F, Kaynak D. An evaluation of the effects of two distal extension removable partial denture designs on tooth stabilization and periodontal health. $J$ Oral Rehabil 2005 32; 823-829.

16. Mahmood wa, salim sa, saharudin s. the status of the abutment teeth in distal extension removable partial dentures. Malay Dent J 200930 ;(1) 13-19.

17. Polly S. Ma, Brudvik JS. Managing the maxillary partially edentulous patient with extensive anterior tooth loss and advanced periodontal disease using a removable partial denture: A clinical report. J Prosthet Dent 2008; 100:259-263.

18. Berg E. Retención de las prótesis totales. En: Thorén MM, Gunne J. Prótesis Removible. AMOLCA, Actualidades Médicas, CA; 2014; 133-138.

19. Wada S, Wakabayashi N, Tanaka T, Ohyama T. Influence of abutment selection in maxillary Kennedy Class II RPD on elastic stress distribution in oral mucosa: an FEM study. J Prosthodont. 2006; 15:89-94

20. Kilfeather GP, Lynch CD, Sloan AJ, Youngson CC. Quality of communication and master impressions for fabrication of cobalt chromium removable partial dentures in general practice in England, Ireland and Wales in 2009. J Oral Rehabil. 2010. 37:300-305.

21. Vanzeveren C, D'hoore W, Bercy P, Leloup G. Treatment with removable partial dentures: a longitudinal study. Part I. J Oral Rehabil 2003; 30 447-458. 